OPEN

SUBJECT AREAS:

ELECTRICAL AND

ELECTRONIC

ENGINEERING

NANOPARTICLES

APPLIED PHYSICS

SENSORS AND BIOSENSORS

Received

1 May 2013

Accepted

5 June 2013

Published

21 June 2013

Correspondence and requests for materials should be addressed to K.F. (fukuda@yz. yamagata-u.ac.jp)

\section{Strain sensitivity and durability in p-type and n-type organic thin-film transistors with printed silver electrodes}

\author{
Kenjiro Fukuda ${ }^{1,2}$, Kenta Hikichi ${ }^{1,2}$, Tomohito Sekine ${ }^{1,2}$, Yasunori Takeda ${ }^{1,2}$, Tsukuru Minamiki ${ }^{1,2}$, \\ Daisuke Kumaki ${ }^{1,2}$ \& Shizuo Tokito ${ }^{1,2}$
}

'Graduate School of Science and Engineering, Yamagata University, 4-3-16 Jonan, Yonezawa, Yamagata, 992-8510, Japan,

${ }^{2}$ Research Center for Organic Electronics, Yamagata University.

Mechanical flexibility and compatibility of printing processes are key advantage that organic electronic devices have over conventional inorganic devices. However, one of the major remaining issues for organic devices is insufficient mechanical durability of printed electrodes. Here we have investigated the mechanical durability of both p-type and n-type organic thin-film transistors (TFTs) with ink-jet printed silver electrodes from silver nanoparticle inks. The modified silver nanoparticle inks enabled the strong adhesion to the underlying polymer layer, and the fabricated organic TFTs exhibited excellent reproducibility in the bending cycle tests. The strong channel length dependence on the strain sensitivity was observed in both p-type and n-type organic TFTs. The organic TFTs with a short-channel exhibited higher sensitivity to the bending strain. These results suggest that the flexible organic TFTs with printed silver electrodes have excellent mechanical durability and are useful for bending and strain sensors.

M echanical flexibility is a key advantage that organic electronic devices have over conventional inorganic devices. Organic materials intrinsically have mechanical flexibility due to Van der Waals bonding between organic molecules, making ultra-flexible organic devices feasible ${ }^{1,2}$. Besides the flexibility, compatibility of printing processes is another key advantage of the organic devices. Fabrication processes based on printing have a number of advantages over conventional photolithography. Printable processes can dramatically reduce material waste and manufacturing process steps while lowering manufacturing costs. Moreover, they can be readily scaled to large-area production with high throughput. These features enable low-cost, large-area, and flexible device applications, leading to new ways to develop organic electronics in areas such as lightemitting-diodes ${ }^{3}$, solar cells ${ }^{4}$, thin-film transistors $(\mathrm{TFTs})^{1,5,6}$, logic $_{\text {circuits }}{ }^{7-9}$, and sensors ${ }^{10-12}$.

The effect of compressive or tensile strain on the performance of organic TFT devices was initially reported by Sekitani et al. ${ }^{1,13,14}$, followed by other groups ${ }^{2,15-20}$. The authors demonstrated that compressive strain led to an increase while tensile strain led to a decrease in the mobility of organic TFT devices beyond that expected solely from change in the capacitance of the dielectric layer. Recent studies have explored the relationship between the behavior of organic TFT devices under strain and the various factors in the organic TFT devices such as molecular structures of semiconducting materials ${ }^{15,16,19,20}$, dielectric materials and interfaces between dielectric layers and semiconductor layers ${ }^{18}$, or contact resistance between source and drain electrodes of organic TFT devices ${ }^{17}$. However, the gate, source, and drain electrodes used in all previous studies were evaporated metals. To achieve flexible organic electronics based on organic TFT devices fabricated by full solution processes, the electrical characteristics must be reproducible in repetition in bending. Reproducible change in on-current in organic TFT device can also be applied to thin-film bending or strain sensors.

Many research groups have studied solution-processed organic TFT devices using various printing methods such as ink-jet ${ }^{7,21-23}$, aerosol-jet ${ }^{24,25}$, screen ${ }^{26}$, flexo ${ }^{27}$, and gravure printing ${ }^{8,28,29}$. Among them, the fabrication of electrodes from silver nanoparticle inks through inkjet printing process has been the most reported for printed organic TFT devices ${ }^{7,21}$ because this enables thin and highly conductive electrodes that can reduce operational voltages of printed devices ${ }^{30}$. However, in contrast to their advantages in terms of high conductivity and low sintering temperature, the printed electrodes from silver nanoparticle ink have a problem in terms of mechanical durability because of a lack of adhesion strength on the substrates ${ }^{31}$. 
In this study, we have fabricated both p-type and n-type organic TFT devices with inkjet-printed silver electrodes from a silver nanoparticle ink and studied the mechanical durability of those devices under tensile strain. By using modified silver nanoparticle ink, we achieved the strong fusion of the printed silver electrodes with the underlying polymer layers, resulting in excellent mechanical durability against the bending strain. The on-current of the organic TFT device clearly decreased as tensile strain increased, which is reproducible even in a bending radius of $4 \mathrm{~mm}$. We found that the change in on-current can be significantly enhanced in shorter channels.

\section{Results}

Flexible p-type and n-type organic TFT devices with printed electrodes. Figure 1a schematically shows the structure of the fabricated devices with bottom-gate bottom-contact configurations. Our study dealt with both p-type and n-type organic semiconductor active materials: namely, pentacene as p-type and a benzobis (thiadiazole) (BBT) derivative with trifluoromethylphenyl groups (FPTBBT) as an n-type semiconducting material (figure $1 \mathrm{~b})^{32,33}$. The materials were chosen owing to their relatively high hole and electron mobility. Moreover, pentacene was a standard material for the p-type organic semiconductor, and the effects of strain have been well studied by several other groups ${ }^{1,13,14,16,18,20}$. Thermally crosslinked poly(4-vinyl-phenol) (PVP) was used as the dielectric material. The inkjet-printed silver electrodes from silver nanoparticles dissolved in water-based solvent were used as gate, source, and drain electrodes. Photographs of the fabricated devices are shown in figure $1 \mathrm{c}, 1 \mathrm{~d}$, and $1 \mathrm{e}$. We can obtain fine source/drain electrodes with line width of $25 \mu \mathrm{m}$ as shown in Figure 1e.

Adhesion of silver electrodes on the polymer substrates. The adhesion of printed silver electrodes to the underlying cross-linked PVP layers was evaluated by scanning electron microscopy (SEM). The SEM image of printed silver electrodes is shown in figure $2 \mathrm{a}$. There is no clearance gap between silver electrodes and cross-linked PVP layers, indicating that the printed silver electrodes obtained from the silver nanoparticles exhibited the strong adhesion on polymer layers. Indeed, no cracking/buckling of silver electrodes was observed even after outward bending with a bending radius of $4 \mathrm{~mm}$ (1.6\% tensile strain), and the electrodes exhibited almost the same surface after strong tensile strain (figure $2 b$ and $2 c$ ). In contrast,

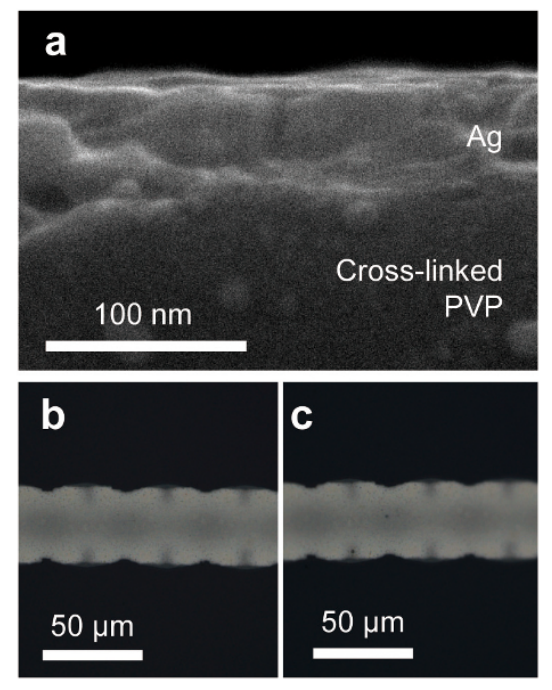

Figure $2 \mid$ Adhesive of the printed silver electrodes on polymer substrates. (a) Cross sectional SEM image of interface between printed silver electrodes and cross-linked PVP base layer. Photographs of printed silver source electrodes (b) before and (c) after the application of $1.6 \%$ tensile strain $(R=4.0 \mathrm{~mm})$.

the silver electrodes obtained from other silver nanoparticles dissolved in a tetradecane (NPS-JL, Harima chemicals) did not exhibit such a strong adhesion property (see supporting Fig. S2). Therefore, the strong durability against the mechanical strain was attributed to the composition of the silver nanoparticle ink used. Indeed, the silver nanoparticle ink used in this study contained $1-5 \mathrm{wt} \%$ synthetic resin, which might play an important role as an interfacial binder to the polymer dielectric layer.

Transistor performance. Figure 3 shows the transfer characteristics of both p-type and n-type devices before bending experiments. The source-drain current $\left(I_{D S}\right)$ was measured as a function of the gatesource voltage $\left(V_{G S}\right)$ at a constant drain-source voltage $\left(V_{D S}\right)$ in a nitrogen atmosphere. $V_{D S}$ was set to $-20 \mathrm{~V}$ for a p-type devices and $+20 \mathrm{~V}$ for n-type devices. Both pentacene and FPTBBT transistors a

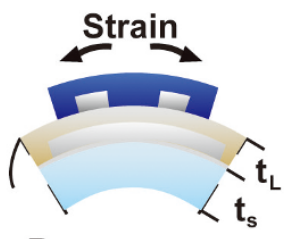

$\mathbf{R}$

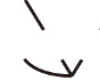

C

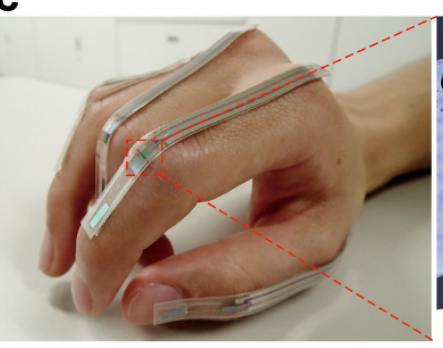

b

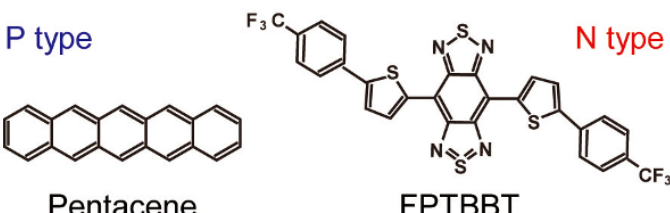

Pentacene
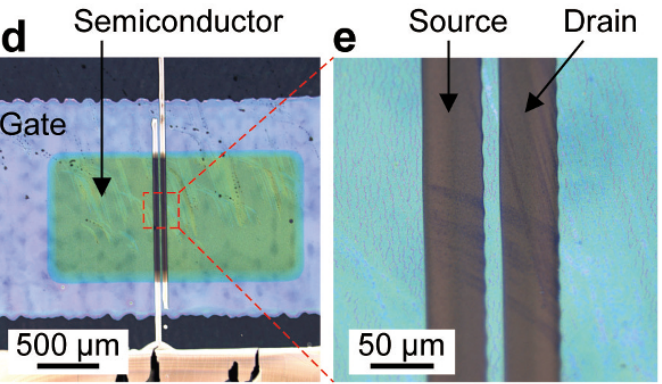

Figure $1 \mid$ Flexible organic TFT devices with printed electrodes. (a) Illustration of an organic TFT device with inkjet-printed silver electrodes under tensile strain. (b) Chemical structure of organic semiconducting materials used in this study. Pentacene as p-type (left) and FPTBBT as n-type (right). (c) A Photograph of the fabricated organic TFT devices installed on human fingers. (d) Optical microscope image of a fabricated TFT and (e) magnified image for channel region. 


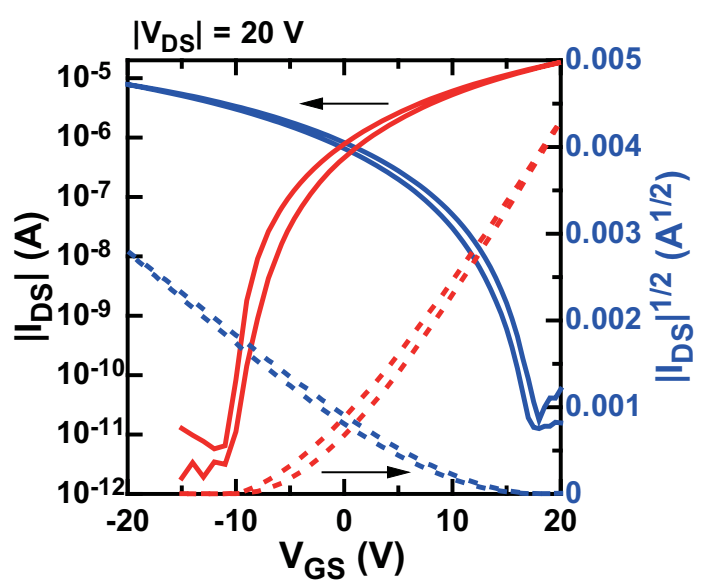

Figure 3 Transistor characteristics of both p- and n-type organic TFT devices without strain. Transfer characteristics of both pentacene-based (blue) and FPTBBT-based (red) organic TFT devices before application of tensile strain. Both p-type and n-type TFTs have almost equal channel length of $\sim 10 \mu \mathrm{m}$. Applied source-drain voltages $\left(V_{D S}\right)$ for pentacene- and FPTBBT-based devices were $-20 \mathrm{~V}$ and $+20 \mathrm{~V}$, respectively.

with printed electrodes exhibited good electrical performances. The estimated field-effect mobility $(\mu)$ in the saturation region was $0.12 \mathrm{~cm}^{2} \mathrm{~V}^{-1} \mathrm{~s}^{-1}$ for both p-type and n-type transistors, and on/off ratio was greater than $10^{5}$. The typical device performances of both $\mathrm{p}$ type and n-type TFT devices in the absence of strain is summarized in Table 1.

Effect of strain on device performance. In this study, the tensile strains were applied to the organic TFT devices with printed silver electrodes. The strains were parallel to the source-drain current paths, and the performances of organic TFT devices were evaluated before, during, and after the application of strains. The surface strain $(S)$ induced on the active layer was evaluated using the following formula ${ }^{34}$ :

$$
S=\left(\frac{d_{t}+d_{s}}{2 R}\right) \frac{\left(1+2 \eta+\chi \eta^{2}\right)}{(1+\eta)(1+\chi \eta)}
$$

In which $d_{t}$ and $\mathrm{d}_{\mathrm{s}}$ are the thicknesses of the active layer and the substrate, respectively, $\eta$ is $d_{t} / d_{s}, \chi$ is the ratio between the Young moduli of the active layer and of the substrate $\left(\chi=Y_{l} / Y_{s}\right)$, and $\mathrm{R}$ is the bending radius. Young's modulus of the PEN substrate is $12.2 \mathrm{GPa}$, and the $\chi$ is estimated 1.6. Considering that the thickness of substrate $(125 \mu \mathrm{m})$ is more than two orders of magnitude thicker than the active layer $(<1 \mu \mathrm{m})$, the surface strain induced on the active layer can be approximated by:

$$
S=\frac{d_{s}}{2 R}
$$

We applied a bending strain with radii ranging from $11 \mathrm{~mm}$ down to $4 \mathrm{~mm}$, corresponding to an induced surface strain from $0.57 \%$ to $1.6 \%$. All the measurements were carried out from the small deformation regime (large bending radii), and the deformation was constantly increased by reducing the bending radius. The reversibility of the device behavior was checked by measuring the

Table 1 Summary of typical device performance of both pentacene- and FPTBBT-based TFT devices with channel length of $10 \mu \mathrm{m}$

\begin{tabular}{lccc} 
Semiconductor & $\mu\left(\mathrm{cm}^{2} \mathrm{~V}^{-1} \mathrm{~s}^{-1}\right)$ & $V_{T H}(\mathrm{~V})$ & On/Off \\
\hline Pentacene & 0.12 & 6.4 & $5.6 \times 10^{5}$ \\
FPTBBT & 0.12 & -0.9 & $1.0 \times 10^{7}$ \\
\hline
\end{tabular}

electrical characteristics of the devices in the flat position after each deformation step. For each summary plot, at least two separate devices were tested to ensure reproducibility of the device characteristics.

The electrical behavior of the flexible devices was characterized during the systematic application of tensile strain. Figure 4 shows representative examples of the transfer characteristics in observed electrical characteristics for pentacene and FPTBBT-based devices on cross-linked PVP with channel length of $10 \mu \mathrm{m}$. Gate voltage $\mathrm{V}_{\mathrm{GS}}$ is swept from 20 to $-20 \mathrm{~V}$ with the application of $\mathrm{V}_{\mathrm{DS}}=-20 \mathrm{~V}$ for p-type devices (figure $4 \mathrm{a}$ ), and $\mathrm{V}_{\mathrm{GS}}$ is swept from -15 to $20 \mathrm{~V}$ with the application of $\mathrm{V}_{\mathrm{DS}}=20 \mathrm{~V}$ for n-type devices (figure $4 \mathrm{c}$ ). In agreement with previous reports, both $\mathrm{p}$ - and n-type devices on polymer dielectrics exhibited a decrease in saturation on-current $\mathrm{I}_{\mathrm{DS}}$ upon the application of tensile strain ${ }^{13,18,19}$. The changes in the on-current are $-31 \%$ for a pentacene-based device and $-30 \%$ for a FPTBBT-based device at $1.6 \%$ tensile strain. We observed a relatively large hysteresis in the transfer characteristics while applying tensile strain, especially in the short channel region. Although the hysteresis became larger during the application of large strain, the transfer characteristics completely returned to the initial state after the tensile strain. Such reproducibility in the device characteristics was also observed for both pentacene and FPTBBT-based devices (Figure 4b and $4 d$ ). The on-current $I_{D S}$ value was normalized with initial $I_{D S}$ with application of no strain. The on-current $I_{D S}$ decreased as application of tensile strain was increased, and the value returned to its initial value when the application of strain stopped even after the $1.6 \%$ strain $(R=4.0 \mathrm{~mm})$. The mechanical flexibility and reproducibility were comparable with previously reported organic TFT devices with evaporated $\mathrm{Au}$ electrode ${ }^{13}$. The excellent mechanical flexibility of our TFT devices with printed silver electrodes is attributed to the strong fusion of the electrodes with the underlying dielectric surfaces. Therefore, these results exhibited the feasibility of applying inkjet-printed electrodes from nanoparticle inks to the flexible electronics.

Structural dependence on the strain sensitivity of organic TFT devices. Furthermore, the electrical characterization was performed for devices with different channel width $(W)$ and channel length $(L)$. The channel width dependence is shown in figure $5 \mathrm{a}$, and the channel length dependence in figure $5 b$ and $5 c$. The normalized on-current of pentacene-based devices with the same channel length of $70 \mu \mathrm{m}$ and with three different channel widths $(300,1000$, and $3000 \mu \mathrm{m})$ are plotted as a function of tensile strain in figure $5 \mathrm{a}$. The normalized on-current of pentacene-based devices with the same channel width of $1000 \mu \mathrm{m}$ and with three different channel lengths $(150,70$, and $10 \mu \mathrm{m})$ are plotted as a function of tensile strain in figure $5 \mathrm{~b}$, and the same plot of FPTBBT-based devices is shown in figure $5 \mathrm{c}$. Channel width and on-current variation do not correlate, which agrees with the models that explain the current change in organic TFT devices under application of $\operatorname{strain}^{34}$. On the other hand, the strain sensitivity clearly depends on the channel length for both pentacene- and FPTBBTbased devices. As the channel length shortened, the current became more sensitive to the surface strain. The application of $1.6 \%$ tensile strain to FPTBBT-based devices with channel lengths of $10 \mu \mathrm{m}$ and $150 \mu \mathrm{m}$ results in $31 \%$ and $18 \%$ changes in the on-current, respectively (Fig. 5b). The same tendency was observed for FPTBBT-based devices. The application of $1.6 \%$ tensile strain to FPTBBT-based devices with channel lengths of $10 \mu \mathrm{m}$ and $150 \mu \mathrm{m}$ results in a $30 \%$ and $14 \%$ changes in the on-current, respectively (Fig. 5 c). The printed devices exhibited strong channel length dependence on strain sensitivity, which has not been previously reported. The current change is linearly proportional to the application of surface strain for each channel length and each semiconductor material. For each curve, we have also estimated the slope of the sensitivity curve 


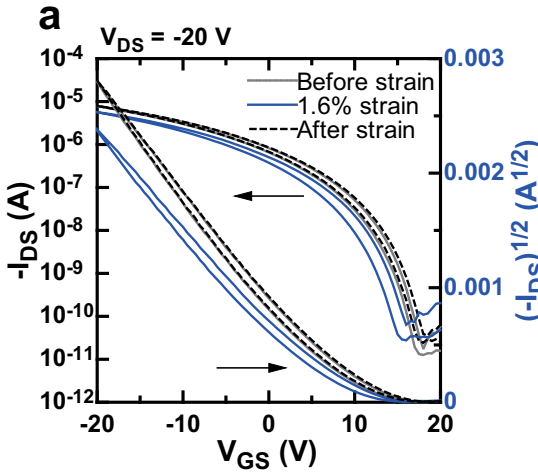

b
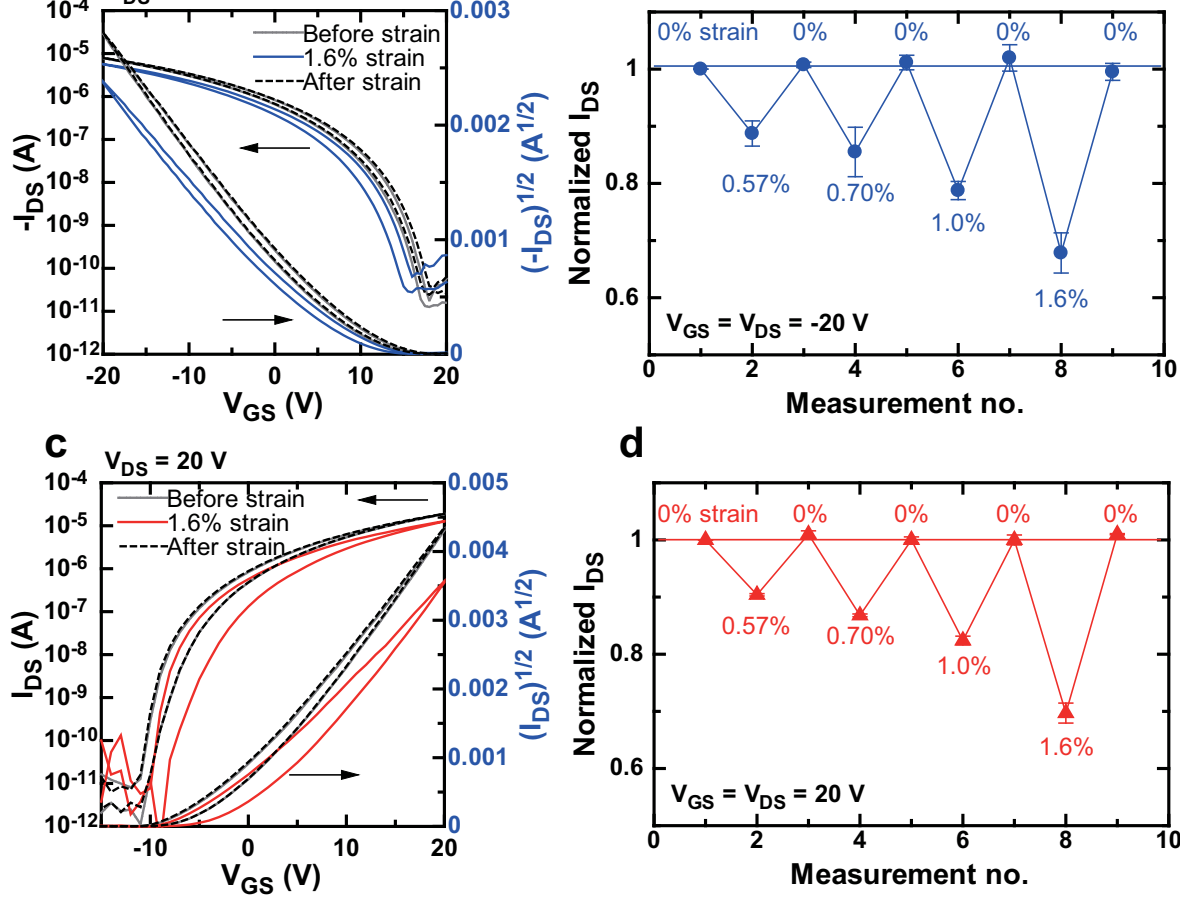

d

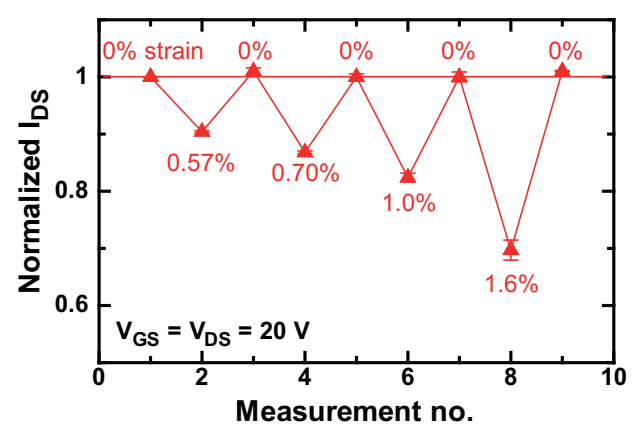

Figure $4 \mid$ Electro-mechanical stability of organic TFT devices before, during, and after bending. (a) Transfer characteristics of the pentacene-based TFT device with channel length of $10 \mu \mathrm{m}$ before, during, and after application of $1.6 \%$ tensile strain. (b) Saturation source-drain current ( $\left.I_{D S}\right)$ of pentacene-based devices monitored at $V_{D S}=V_{G S}=-20 \mathrm{~V}$ as a function of measurement number. I $\mathrm{DS}$ was normalized from its initial value (without application of strain). (c) Transfer characteristics of the FPTBBT-based TFT device with channel length of $10 \mu \mathrm{m}$ before, during, and after application of $1.6 \%$ tensile strain. (b) Saturation source-drain current $\left(I_{D S}\right)$ of pentacene-based devices monitored at $V_{D S}=V_{G S}=+20 \mathrm{~V}$ as a function of measurement number. $\mathrm{I}_{\mathrm{DS}}$ was normalized from its initial value (without application of strain).
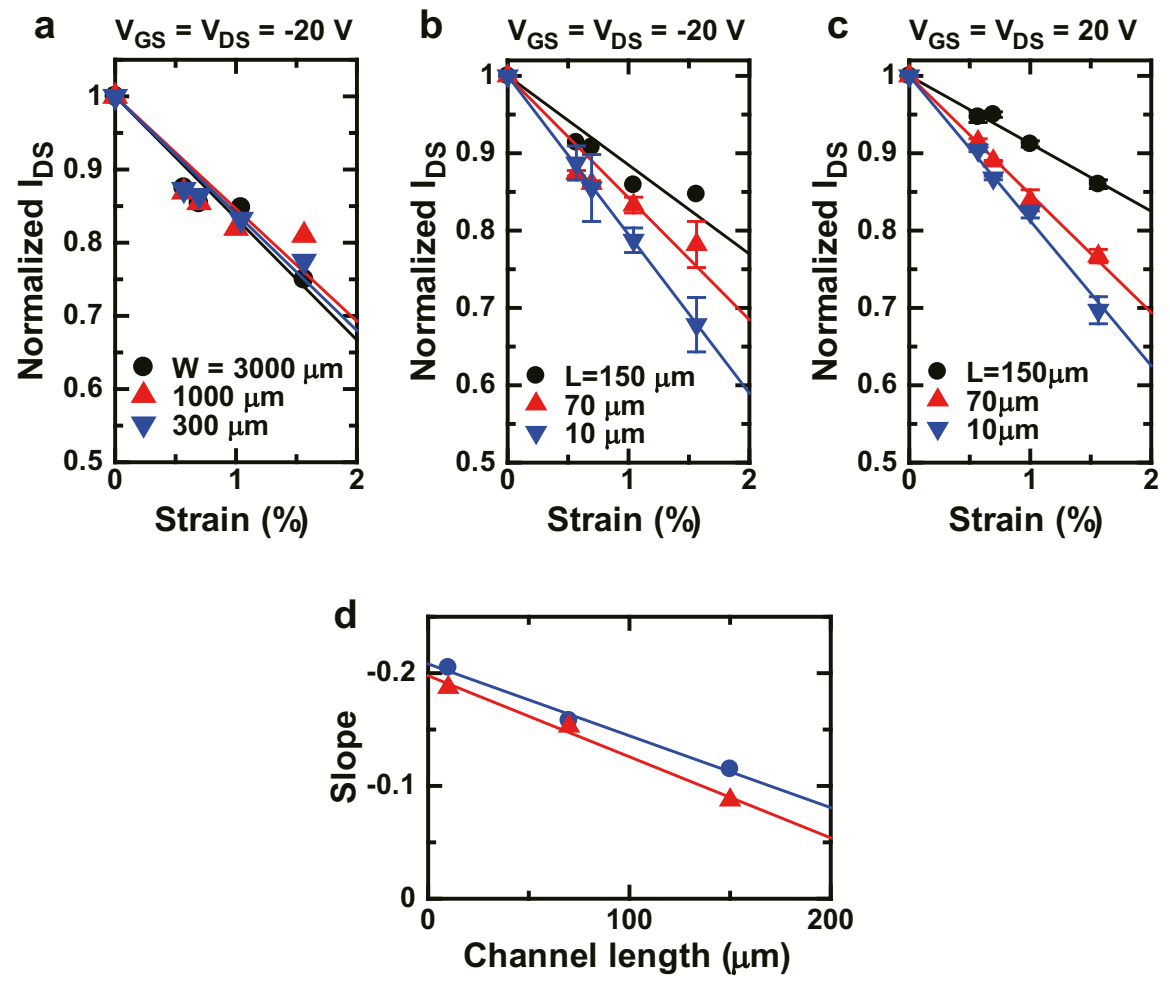

Figure $5 \mid$ Structural dependence on the strain sensitivity of organic TFT devices. (a) Current variation as a function of tensile strain for pentacenebased TFT devices with different channel width. (b) Current variation as a function of tensile strain for pentacene-based TFT devices with different channel lengths. (c) Current variation as a function of tensile strain for FPTBBT-based TFT devices with different channel lengths. (d) Correlation between sensitivity slopes estimated from fitting curves in (b) and (c) and channel lengths of the TFT devices, showing the device sensitivity clearly depended on the channel lengths of both pentacene-based (blue) and FPTBBT-based (red) organic TFT devices. 
and plotted it as a function of the channel length as shown in figure $5 \mathrm{~d}$. There was linear dependence between slope and channel length, at least in the channel length range that we have studied.

\section{Discussion}

To understand the mechanism of channel length dependence on strain sensitivity, we here consider the total resistance $\left(\mathrm{R}_{\mathrm{ON}}\right)$ between source and drain electrodes:

$$
R_{O N}=R_{c h}+R_{C}
$$

in which $R_{c h}$ is a channel resistance and $\mathrm{R}_{\mathrm{C}}$ is a contact resistance. First, we consider the $R_{c h}$, which is the channel region of organic TFT devices. Several recent studies set out to explore the relationship between the semiconductor molecular structure and the behavior of organic TFT devices under strain. Specifically, pentacene grain size dependence on the strain sensitivity was reported by Cosseddu et $\mathrm{al}^{20}$. According to this report, the larger the pentacene grain, the larger the strain sensitivity of the organic TFT devices, which was attributed to the hopping transport of charge carrier in the organic semiconductor films ${ }^{35}$. In our results, although the semiconductor grain sizes in both pentacene and FPTBBT were almost the same in different channel lengths, the tendency of the relationship between the strain sensitivity and the channel length is similar to the experimental results reported by Cosseddu et al. The shorter channel length means the decreased hopping paths between source and drain electrodes. We should also consider the contact resistance. Chen et al. have reported that the $\mathrm{R}_{\mathrm{C}}$ of pentacene-based organic TFT devices increased slightly during the application of tensile strain ${ }^{17}$. For shorter channel length, the $R_{c h}$ decreases and the $R_{C}$ becomes dominant in total resistance. Therefore, the increasing of $R_{C}$ might be a possible mechanism for the on-current change in our organic TFT devices under the tensile strain. We are further studying the mechanism of the strain effect on the on-current in printed organic TFT devices.

In conclusion, we have studied the effect of tensile strain on organic thin-film transistors with printed silver electrodes fabricated on a thin plastic substrate. The printed silver electrodes were fabricated from modified nanoparticle ink by ink-jet printing. The fabricated organic TFT devices exhibited excellent electrical performances, and reproducible strain dependence of the on-current. This mechanical durability is attributed to strong adhesion between the printed silver electrodes and the underlying polymer layers. Moreover, in both p-type and n-type organic semiconductors, we found that channel length of organic TFT devices plays a significant role in determining sensitivity of transistor characteristics to the applied strain. From these experimental results, we concluded that by properly tuning the channel length, the strain sensitivity in both $\mathrm{p}$ type and n-type organic TFT devices can be controlled easily. The devices with a high strain sensitivity can be made useful for sensing applications by using a short channel length. In contrast, wider channel length is suitable for decreasing their sensitivity to strain.

\section{Methods}

Materials. The ink used as printed electrodes in this work was a silver nanoparticle ink in a water-based solvent (JAGLT-01, DIC, Corp. Japan). The ink contained 25-35 $\mathrm{wt} \%$ silver nanoparticles with an average diameter of $20-40 \mathrm{~nm}$ and $1-5 \mathrm{wt} \%$ synthetic resin. A cross-linked poly-4-vinylphenol (PVP) was used as a planarization layer and gate dielectric layers. PVP $(\mathrm{Mw} \sim 25000$, Sigma Aldrich Co. $)$ and poly(melamine-co-formaldehyde) $(\mathrm{Mn} \sim 432,84 \mathrm{wt} \%$, Sigma Aldrich Co.) as a crosslinking agent were mixed in propylene glycol monomethyl ether acetate (PGMEA). The PVP solution was spin-coated onto glass substrates and was thermally crosslinked at temperatures of $150^{\circ} \mathrm{C}$ for $60 \mathrm{~min}$ to form a dielectric layer.

Device fabrication. The devices were fabricated on $125-\mu$ m-thick flexible polyethylene naphthalate substrates (PEN). To reduce the surface roughness, our PEN substrates are coated with an 80 -nm-thick polymer planarization layer, which is deposited by spin-coating and cured at a temperature of $150^{\circ} \mathrm{C}$. The silver nanoparticle ink was patterned with an inkjet printer (Fujifilm Dimatix, DMP2800) onto the planarization layers using a print head with $10 \mathrm{pl}$ nozzles as bottom gate electrodes. The silver nanoparticle ink was printed using a customized waveform. The droplets were deposited with a dot-to-dot spacing of $60 \mu \mathrm{m}$. During the inkjet patterning process, the substrate temperature was maintained at $30^{\circ} \mathrm{C}$. After the printing, the substrates were stored for $30 \mathrm{~min}$ in an environmental test chamber (espec, $\mathrm{SH}-221$ ) in which the temperature was held at $30^{\circ} \mathrm{C}$ and relative humidity was held at $95 \% \mathrm{RH}$ in order to planarize the electrodes ${ }^{30}$. After the drying process, the substrates were heated at $140^{\circ} \mathrm{C}$ for 1 hour to sinter the silver nanoparticles. The fabricated silver gate electrodes had uniform thickness of $\sim 100 \mathrm{~nm}$ (see supporting figure S1). After these electrodes were formed, a solution of cross-linked PVP was spin-coated and baked to form 350-nm-thick dielectric layers. The silver electrodes were subsequently inkjet printed using a print head with $1 \mathrm{pl}$ nozzles to form the bottom source/drain electrodes. The droplets were deposited with a dot-to-dot spacing of $20 \mu \mathrm{m}$. The device geometry was defined by the patterning data of inkjet printing. Finally, a 50-nm-thick pentacene or FPTBBT semiconducting layer was deposited on the source/drain electrodes at a pressure below $5.0 \times 10^{-5} \mathrm{~Pa}$ with a deposition rate of $0.2 \AA / s$. During the deposition, the substrates were not heated nor cooled.

Device characterization. The electrical characteristics of the fabricated TFT devices were measured by using a semiconductor parameter analyzer (Keithley, 4200-SCS). The bending experiments were carried out by wrapping the PEN film around the rod with various radii from 11 to $4 \mathrm{~mm}$. The TFT devices were stressed to the tensile strain parallel to the source/drain electrodes. For each summary plot, at least two separate devices were tested to ensure reproducibility of the device characteristics. All electrical measurements of organic TFT devices were performed in a nitrogen atmosphere to prevent degradation of organic semiconductors induced by oxygen or moisture ${ }^{36}$. The cross-sectional structure of the printed electrodes was examined by a scanning electron microscope (SEM) (JEOL, 7300F).

1. Sekitani, T. et al. Flexible organic transistors and circuits with extreme bending stability. Nature Mater 9, 1015-1022 (2010)

2. Yi, H. T. et al. Ultra-flexible solution-processed organic field-effect transistors. Nature Commun 3, 1259 (2012).

3. Sekitani, T. et al. Stretchable active-matrix organic light-emitting diode display using printable elastic conductors. Nature Mater 8, 494-499 (2009).

4. Kaltenbrunner, M. et al. Ultrathin and lightweight organic solar cells with high flexibility. Nature Commun 3, 770 (2012).

5. Khodagholy, D. et al. In vivo recordings of brain activity using organic transistors. Nature Commun 4, 1575 (2013).

6. Ikawa. et al. Simple push coating of polymer thin-film transistors. Nature Commun 3, 1176 (2012).

7. Ng, T. N. et al. Scalable printed electronics: an organic decoder addressing ferroelectric non-volatile memory. Sci. Rep 2, 585 (2012)

8. Jung, M. et al. All-Printed and Roll-to-Roll-Printable 13.56-MHz-Operated 1-bit RF Tag on Plastic Foils. IEEE Trans. Electron. Devices 57, 517-580 (2010).

9. Myny, K. et al. Organic RFID transponder chip with data rate compatible with electronic product coding. Org. Electron 11, 1176-1179 (2010).

10. Maiwald, M., Werner, C., Zoellmer, V. \& Busse, M. Sens. Actuators, A 162, 198-201 (2010)

11. Jang, J., Ha, J. \& Cho, J. Fabrication of Water-Dispersible Polyaniline-Poly(4styrenesulfonate) Nanoparticles For Inkjet-Printed Chemical-Sensor Applications. Adv. Mater 19, 1772-1775 (2007).

12. Lipomi, D. J. et al. Skin-like pressure and strain sensors based on transparent elastic films of carbon nanotubes. Nature Nanotechnol 6, 788-792 (2011)

13. Sekitani, T. et al. Bending experiment on pentacene field-effect transistors on plastic films. Appl. Phys. Lett. 86, 073511 (2005).

14. Sekitani, T. et al. Ultraflexible organic field-effect transistors embedded at neutral strain position. Appl. Phys. Lett. 87, 173502 (2005).

15. Yang, C. et al. Bending-stress-driven phase transitions in pentacene thin films for flexible organic field-effect transistors. Appl. Phys. Lett. 92, 243305 (2008).

16. Jedaa, A. \& Halik, M. Toward strain resistant flexible organic thin film transistors. Appl. Phys. Lett. 95, 103309 (2009).

17. Chen, F.-C. et al. Influence of mechanical strain on the electrical properties of flexible organic thin-film transistors. Semicond. Sci. Technol. 26, 034005 (2011).

18. Sokolov, A. N. et al. Mechanistic Considerations of Bending-Strain Effectswithin Organic Semiconductors on Polymer Dielectrics. Adv. Funct. Mater. 22, 175-183 (2012).

19. Niga, A. et al. Strain induced anisotropic effect on electron mobility in C60 based organic field effect transistors. Appl. Phys. Lett. 101, 083305 (2012).

20. Cosseddu, P. et al. Continuous tuning of the mechanical sensitivity of Pentacene OTFTs on flexible substrates: From strain sensors to deformable transistors. Org. Electron. 14, 206-211 (2013).

21. Sekitani, T. et al. Organic transistors manufactured using inkjet technology with subfemtoliter accuracy. Proc. Natl. Anal. Sci. 105, 4976-4980 (2008).

22. Sirringhaus, H. et al. High-resolution inkjet printing of all-polymer transistor circuits. Science 290, 2123-2126 (2009).

23. Minemawari, H. et al. Inkjet printing of single-crystal films. Nature 475, 364-367 (2011).

24. Cho, J. H. et al. Printable ion-gel gate dielectrics for low-voltage polymer thin-film transistors on plastic. Nature Mater 7, 900-906 (2008) 
25. Ha, M. et al. Printed, Sub-3V Digital Circuits on Plastic from Aqueous Carbon Nanotube Inks. ACS Nano 4, 4388-4395 (2010).

26. Noguchi, Y. et al. Organic-transistor-based flexible pressure sensors using ink-jetprinted electrodes and gate dielectric layers. Appl. Phys. Lett. 89, 253507 (2006).

27. Yan, H. et al. A high-mobility electron-transporting polymer for printed transistors. Nature 457, 679-686 (2009).

28. Kang, H. et al. High-Performance Printed Transistors Realized Using Femtoliter Gravure-Printed Sub-10 $\mu \mathrm{m}$ Metallic Nanoparticle Patterns and Highly Uniform Polymer Dielectric and Semiconductor Layers. Adv. Mater. 24, 3065-3069 (2012).

29. Vornbrock, A. d. 1. F. et al. Fully gravure and ink-jet printed high speed pBTTT organic thin film transistors. Org. Electron. 11, 2037-2044 (2011).

30. Fukuda, K. et al. Profile Control of Inkjet Printed Silver Electrodes and Their Application to Organic Transistors. ACS Appl. Mater. Interfaces 5, 3916-3920 (2013).

31. Joo, S. \& Baldwin, D. F. Adhesion mechanisms of nanoparticle silver to substrate materials: identification. Nanotechnology 21, 055204 (2010).

32. Kono, T. et al. Dithienylbenzobis(thiadiazole) based organic semiconductors with low LUMO levels and narrow energy gaps. Chem. Commun. 46, 3265-3267 (2010).

33. Fujisaki, Y. et al. Air-stable n-type organic thin-film transistor array and high gain complementary inverter on flexible substrate. Appl. Phys. Lett. 97, 133303 (2010).

34. Gleskova, H. et al. Electrical response of amorphous silicon thin-film transistors under mechanical strain. J. Appl. Phys. 92, 6224-6229 (2002).

35. Vissenberg, M. C. J. \& Matters, M. Theory of the field-effect mobility in amorphous organic transistors. Phys. Rev. B 57, 12964-12967 (1998).

36. Ashimine, T. et al. Air Stability of p-Channel Organic Field-Effect Transistors Based on Oligo-p-phenylenevinylene Derivatives. Jpn. J. Appl. Phys. 47, 1760-1762 (2008)

\section{Acknowledgements}

We gratefully acknowledge the Japan Science and Technology Agency (JST) for their support in this work. We also thank DIC Corporation for providing silver nanoparticle ink and Ube industries, Ltd. for providing FPTBBT. The authors also express their gratitude to Dr. Takuya Morimoto for his contributions.

\section{Author contributions}

K.F., D.K. and S.T. conceived the printed organic TFT concept, processing, and structure details. K.F. constructed and characterized the organic TFT devices. K.H., Y.T. and T.M. assisted with device fabrication and characterization. T.S. conducted the SEM observation. K.F. and S.T. wrote the manuscript. K.F. prepared the figures. S.T. supervised the project.

\section{Additional information}

Supplementary information accompanies this paper at http://www.nature.com/ scientificreports

Competing financial interests: The authors declare no competing financial interests.

How to cite this article: Fukuda, K. et al. Strain sensitivity and durability in p-type and n-type organic thin-film transistors with printed silver electrodes. Sci. Rep. 3, 2048; DOI:10.1038/srep02048 (2013).

(c) (i) $\Theta$ This work is licensed under a Creative Commons AttributionBY NC ND NonCommercial-NoDerivs 3.0 Unported license. To view a copy of this license, visit http://creativecommons.org/licenses/by-nc-nd/3.0 